\title{
Collapse, Crushing and Energy Absorption of Circular-Celled Honeycombs
}

\author{
Jaeung Chung*and Anthony M. Waas, \\ Composite Structures Laboratory, \\ Department of Aerospace Engineering, \\ University of Michigan, Ann Arbor, MI, 48109-2140
}

\begin{abstract}
The crushing response of polycarbonate circular cell honeycomb to inplane uniaxial loading under displacement control is analyzed through a combination of experiment and numerical simulation. The experiments corresonding to two different uniaxial loading conditions are performed using honeycomb material which has a nearly periodic microstructure. In the initial part of the response, the material deforms in a uniform fashion. Next, a nonlinear phase characterized by progressive localization of deformation is observed. The progressive localization causes the walls of each cell to contact. These experimental results are simulated through numerical analysis using the finite element method.
\end{abstract}

\section{Introduction}

Crashworthiness is now routinely used as a design consideration in military aircraft and racing cars. With the advent of using lightweight materials and structures in the automotive and commercial aerospace industry, this aspect is also being studied in these arenas to ensure occupant safety while simultaneously improving structural concepts. For situations that dictate a design based on maximum deformation upto a specified limit, sandwich panels with honeycomb cores offers a viable and efficient means to arrive at a useful structural design. As a first step to understanding failure mechanisms of such panels, we have studied the crushing response of a circular cell honeycomb core when subjected to inplane compressive loading. A natural follow-on to this investigation would be to study sandwich panels made of such cores.

\footnotetext{
* Ph.D candidate.

† Associate Professor of Aerospace Engineering, Associate Fellow AIAA.

${ }^{0}$ Copyright (C) 1999 by Jaeung Chung. Published by the American Institute of Aeronautics and Astronautics, Inc. by permission.
}

The mechanical properties of cellular materials were initially studied by Gent and Thomas[1]. Gibson and co-workers[2] calculated the inplane Young's moduli and Poisson's ratios of hexagonal honeycombs for loading in two orthogonal directions. Silva, Hayes and Gibson[3], Klintworth and Stronge[4] and Gibson and Ashby[5,6] have also studied the mechanical response of honeycombs. Papka and Kyriakides[7] studied the loaddisplacement response under displcement control using hexagonal aluminum honeycomb specimens. These researchers also investigated the mechanical response of circular celled honeycomb under uniaxial loading in one direction and also the response to biaxial loading using a specially designed biaxial loading fuxture[8], while Grenestedt[9] carried out a study on the effects of wall waviness on the elastic response of honeycombs.

In the present paper, we have studied the compressive behavior of two dimensional circular cell honeycombs made of polycarbonate, subject to two different inplane loading conditions through an experiment and a corresponding numerical analysis. The objective of the present study is to identify features of the compressive failure mechanism of these solids with the eventual goal of providing an understanding that will enable modeling of these materials as equivalent continua, which reflect the observed experimental results.

\section{Experiment}

Two types of experiments were conducted on the honeycomb specimens which were subjected to uniaxial compressive loading. The first experiment was aimed at examining the collapse mechanism of the honeycomb material under uniaxial, compressive load in the Y-direction (see Figure 1 for nomenclature). The other was aimed at studying the collapse mechanism of the honeycomb material under uniax- 
ial compressive load in the X-direction (Figure 1). The Young's modulus of the polycarbonate material used in the study is $2.422 \mathrm{GPa}$, and the Poisson's ratio is 0.3 . The stress-strain curve for the polycarbonate material that is used to manufacture the honeycomb is shown in Figure $\mathbf{2}^{\mathbf{1}}$. In theory, a hexagonally packed circular cell honeycomb must be transversely isotropic (the X-Y plane being the plane of isotropy), but in practice, due to various imperfections as noted later, these materials are best classified as being orthotropic.

\subsection{Details of Specimens}

The dimensions of the test specimens are described in Figure 1. The test specimens consist of "twelve by twelve" cells cut from a 30.5 by $30.5 \mathrm{~cm}^{2}$ sheet of honeycomb. Using an optical microscope, detailed dimensions of cells in the honeycomb specimens such as cell wall thickness and "radius" of cell were measured accurately. The shape of each cell in the test specimen is not perfectly circular, but deviates slightly from circularity. The distribution of cell aspect ratio, defined as the ratio of the cell diameter in the X-direction divided by the cell diameter in the Y-direction for each cell of the specimen is shown in Figure 3. Also, Figure 3 shows the mean values of " $\mathrm{a}$ " (the diameter in the $\mathrm{X}$-direction), " $\mathrm{b}$ " (the diameter in the Y-direction) and " $\mathrm{t}$ " (single wall thickness) obtained through an optical microscope. In addition, the wall thickness of a polycarbonate honeycomb specimen varies along each cell wall. The values of single wall thickness were found to be $0.066 \pm 0.024 \mathrm{~mm}$ and the double wall thickness corresponding to the region of contact between the neighboring cells, to be $0.143 \pm 0.020 \mathrm{~mm}$.

\subsection{Experimental Method}

Compression experiments were conducted under displacement control in a hydraulic four actuator serve controlled test frame. The overall experimental setup used is shown schematically in Figure 4. Honeycomb specimens were placed between an upper and a lower solid steel plate which are hardened, ground and have lubricated surfaces. The upper and the lower loading plates are mounted on the upper and lower actuators of the test frame respectively. The upper actuator and the lower actuator of the test frame move at the same time collinearly in opposite directions. To record the specimen axial shortening measured through an LVDT and the

\footnotetext{
${ }^{1}$ Supplied by Prof. S. Kyriakides, Univ. of Texas at Austin, for which we are very grateful.
}

specimen load measured through a load cell installed on the upper loading plate, an inhouse data acquisition system was used. The measured loads are normalized by the undeformed effective cross-sectional area and the specimen axial shortening is normalized by the undeformed height of the specimens. These measures of effective stress and normalized axial end shortening are used for presentation of experimental results and subsequent comparison with numerical prediction. Using this same experimental setup, the biaxial compressive behavior of the honeycombs were also studied and are reported elsewhere[10].

\section{Numerical Analysis}

The response of the honeycomb was studied via the finite element method. The commercial code Abaqus was used for this purpose. In the Finite Element Analysis (FEA), a model was made of the entire microsection of the honeycomb (Figure 2) using averaged measured representative data as recorded via an optical microscope. The model includes wall thickness variations and cell ellipticity (Figure 3). The procedure to generate the FEA mesh for the 12 by 12 cell microsection was as follows: Cell wall thickness variation and cell ellipticity was painstakingly measured for several 12 by 12 cell specimens. The data was gathered and analyzed to obtain average cell ellipticity, average cell wall thickness variation, average double wall thickness(occuring at those locations where two cells meet) and average contact area. Next, a unit representative cell(URC) was meshed that include these average measured properties. After this, the unit cell was periodically extended in the horizontal and vertical directions to generate the entire 12 by 12 cell model of the test specimen. Thus, the 12 by 12 FEA mesh is periodic, with the URC representing the average values of imperfection and cell ellipticity as recorded through the measurements carried out via an optical microscope. The URC is discretized with three noded quadratic beann elements. This beam element (B22) is based on Timoshenko beam theory and includes transverse shear deformation. The transverse shear deformation in this element is treated as if the response were linear elastic, independent of the axial and bending responses, even when it is used for the purpose of analyzing nonlinear geometric and nonlinear material behavior. Each cell of the honeycomb is modeled with 20 curved beam elements, and the contact region between neighboring cells is made of 2 beam elements. To include thickness variation, each beam element in the model has a differ- 
ent thickness that is representative of what was measured through the optical microscope. To prevent penetration among cells during deformation, contact elements were included. Using rigid bar elements to simulate the loading plates, the model is loaded in a displacement-controlled manner along the $\mathrm{X}$ direction and along the $\mathrm{Y}$-direction, respectively. In the FEA, the honeycomb material is modeled as a small strain J2 incremental theory of plasticity solid. The uniaxial stress vs. strain response of the polycarbonate used for this purpose is shown in Figure 2.

\section{Results}

Several polycarbonate specimens were tested using the experimental procedure described above. Their responses were simulated through numerical analysis using the finite element method. Typical compressive responses of the specimens under two different uniaxial loading conditions are as shown in Figure 5 and Figure 6. The experimental and numerical findings are explained as follows.

\subsection{Compressive Loading in the $\mathrm{X}$ - direction}

The experimental and numerical response of a honeycomb specimen under a compressive loading in the $\mathrm{X}$-direction is shown in Figure 5 . Figure 5 also shows a sequence of deformed configurations of the specimen in the experiment and the simulation, respectively. In Figure 5, the straight line is the stressnormalized end shortening response as measured in the experiment and the dashed line is the response as obtained via the simulation. In the first phase of the experimental response, the specimen deforms in a uniform fashion (see figure 5-ex1). The 'slope' of the response begins to undergo a slight change around a stress of $20 \mathrm{KPa}$ (see figure 5-ex1). This implies that the response becomes nonlinear due to a reduction in the stiffness of the specimen. In this nonlinear regime, the specimen is 'stable' because the load increment required for further deformation of the specimen is positive. The continuous reduction of the stiffness of the specimen results in a gradually weakening response until the attainment of a maximum load. During this region of the response which is still nonlinear and stable, a cluster of cells shows a tendency to collapse resulting in deformation localization. The initiation of localization is clearly seen in Figure 5-ex4. In Figure 5-ex4, the localization initiates along diagonal lines of the spec- imen, that is, from a cell at the left end top row to a cell at the right end and seventh row from top row and along the other diagonal. This diagonal localization is symmetric about the center line of the specimen in the $\mathrm{X}$ direction. The significant development of the localization results in a negative stiffness of the specimen. In the numerical simulation, severe localization is found to occur along diagonal lines of the specimen (more to the left part of the specimen as shown in Figure 5-sx6). Localization into an ' $\mathrm{X}$-band' shape was formed at the right part of the specimen in the experiment. As seen in Figure 5 , the numerical response shows good agreement with the experimental response. The predicted difference in the maximum load in both cases is within 6 percent of the measured maximum load. Clearly, these differences are to be expected since the numerical model includes a URC with average properties, which is periodically extended, quite different from the variations that are present in that particular test specimen.

\subsection{Compressive Loading in the $\mathrm{Y}$ - direction}

The experimental and numerical responses of a honeycomb specimen under a compressive loading in the Y-direction is shown in Figure 6. Figure 6 also shows a sequence of deformed configurations of the specimen in the experiment and the corresponding sequence in the simulation. In Figure 6, the stright line is the load-displacement response in the experiment and the dashed line is the response as predicted via the simulation. In the first part of the experimental response, the specimen deforms symmetrically about the axis of loading (see Figure 6-ey1). The slope of the response begins to change slightly at $27 \mathrm{KPa}$ (see Figure 6-ey2). This implies that the response becomes nonlinear due to a reduction in the stiffness of the specimen. In this nonlinear phase, the specimen is stable and initiation of deformation localization is observed at the center cells in the first, second, third and fourth row from the bottom row. A continuous reduction in the stiffness of the specimen progressively occurs until the maximum load is reached. In this decreasing stiffness region, the response is still stable. The development of the localization is clearly seen in Fig. 6-ey3. In Figure 6-ey3, the localization is completely developed in four rows from the bottom row and some center cells in the fifth row from the bottom row. In Figure 6-ey4, the localization is completely developed up to the fifth row from the bottom row. As seen in Fig. 6-ey2, Fig. 6-ey3 and Fig. 6-ey4, the regions of collapsing 
cells gradually spreads from row to row. The fully developed localization causes a faster reduction in the stiffness of the specimen beyond the maximum load. In Fig. 6-ey6, the completely collapsed third row deforms significantly more when compared to other rows in which cells are also collapsed. In Fig. 6-ey7, the walls of each cell in the third row contact each other and the corresponding slope of the response is negative. The slope of the response corresponding to Fig. 6-ey8 is positive, while that corresponding to Fig. 6-ey9 is negative. The series of photos shown in Fig. 6-ey7, Fig. 6-ey8 and Fig. 6ey9, reveal progressively increasing ammounts of cell wall contact. When this process occurs, the corresponding stress-normalized end shortening response curve displays an up-down-up oscillatory feature. In other words, whenever each cell completely collapses thereby resulting in a zero local stiffness contribution momentarilly, the slope of the response turns negative and immediately becomes positive as cell contact begins, once again. This mechanism occurs beyond the maximum load. In the numerical simulation, the second row from the bottom of the specimen was more significantly localized when compared to other localized rows (Figure 6-sy5) while, in the experiment, the third row was deformed most significantly among the rows of the specimen (Figure 6-ey5). As seen in Figure 6, the predicted stiffness of the response in the linear region matched the experimental result very well. The difference between the maximum loads corresponding to the two cases is about 15 percent of the experimental value.

\section{Discussion}

The response under compressive loading in the $\mathrm{X}$-direction is somewhat more complex when compared to the response under compressive loading in the Y-direction. The linear stiffness of the response is affected by several factors such as cell size, wall thickness for each cell and deviation from circularity for each cell of the specimen. The difference between the stiffnesses of the linear regions of the responses under $\mathrm{X}$-direction loading and $\mathrm{Y}$-direction loading is mainly caused by the above mentioned unintended imperfections that are present in the specimens. It is therefore prudent to investigate the sensitivity of the specimen stiffness to these different imperfections, the most important being the deviation from circularity of each cell of the specimen. In other words, if each cell of the specimen is perfectly circular, the difference between the stiffnesses of the linear regions of both responses will only be slightly different re- sulting from the non-uniformity in the thickness distributions along the cell wall contact region. Even this would disappear as the specimen is scaled up to include a larger number of cells. However, if the aspect ratio $(a / b)$ of each cell of the specimen is larger than 1 , the stiffness associated with the linear region of the response under uniaxial $\mathrm{X}$-direction loading becomes larger than the corresponding stiffness in the Y-direction. We examined this by analytically deriving expressions for the stiffnesses of a specimen containing ellipsoidal cells. We also evaluated, for purpose of comparison, a closed form solution for the equivalent macroscopic Youngs modulus of a perfectly circular celled hexagonally packed honeycomb, given by Papka and Kyriakides[1998], as

$$
E^{*}=8.329 \frac{E}{\left(1-\nu^{2}\right)}\left(\frac{t}{R}\right)^{3},
$$

where, $t$ is the cell wall thickness, $R$ is the ccll radius, $E$ the youngs modulus of the cell material and $\nu$, the materials possions ratio. The results are tabulated in Table 1. Details of this derivation are presented elsewhere[11]. It is clear from the table of results (Table 1$)$ that positive cell ellipticity $\left(\frac{a}{b}>1\right)$, results in an increase in the $\mathrm{X}$-direction stiffness and a corresponding lowering of the $\mathrm{Y}$-direction stiffness. Thus, it can be argued that cell ellipticity plays an important role in breaking the $\mathrm{X}-\mathrm{Y}$ symmetry that is required of a transversely isotropic material(the $\mathrm{XY}$ plane being the plane of isotropy in the case of perfectly circular cells with neighboring point contact). In addition, the nonuniformity introduced by neighboring cells contacting each other over a finite region as opposed to a point, also leads to imperfect behavior, although, it can be argued that there is no preferential bias for this type of an imperfection to induce deviation from transversely isotropic behavior. That is, if we examine a series of test specimens with $A \times A$ cells, where $A$ is a gradually increasing number, then, statistically one could argue that imperfections caused by non-uniform line contact between neighboring cells (as opposed to point contact), will induce the same degree of departure in the $\mathrm{X}$ and $Y$ direction stiffnesses from the intended equivalent transverse isotropic behavior. On the other hand, cell ellipticity has a preferential bias, with positive ellipticity $\left(\frac{a}{b}>1\right)$ resulting in an increase in the Xdirection stiffness and a corresponding decrease in the $\mathrm{Y}$-direction stiffness.

The results shown in table 2 is a comparison of the perfect unit representative cell (PURC) results and the full scale numerical simulations and experimentally measured linear stiffnesses of the honey- 
combs in the $\mathrm{X}$ and $\mathrm{Y}$ directions. Notice that the PURC results corresponding to the measured maximum cell ellipticity are lower than the full scale numerical simulations and experimental results. However, for the average cell ellipticity, the PURC prediction for $E_{x}^{*}$ is lower than, and for $E_{y}^{*}$ higher than, the corresponding full scale numerical simulations and experimental results. The PURC results correctly predict the trend in breaking the X-Y symmetry of the stiffnesses (induced macroscopic orthotropy due to cell ellipticity). The differences noted above are due to the lack of perfect periodicity in the test specimens as well as in the full scale numerical simulations. Recall that the PURC approach is based on using a small representative section of a larger specimen, thus, its extension to model specimens containing several cells hinges on the assumption of perfect periodicity. Nevertheless, it is able to correctly predict trends reflected in the experimental results and also to bring out the important effect of induced orthotropy due to cell ellipticity. The importance of the induced macroscopic orthotropy is also reflected in the localized deformation patterns obtained earlier. Indeed, the Xdirection reponse and proceeding "diagonal" localized deformation bands are in stark contrast to the Y-direction response and the corresponding "parallel" bellows type localized deformation bands. Thus, if we were to model these honeycombs as equivalent continua, then a representation that involves macroscopic orthotropy is essential to properly capture the observed localized deformation patterns. What we have here is a geometrically induced imperfection at the microscopic level, resulting in an effect that is reflected at the macroscopic level via the equivalent (macroscopic) constitutive description of the solid. The experimental results (Figure 5 and Figure 6) indicate another feature that is related to the observed induced orthotropy of the honeycomb specimens. The maximum "load" carrying capacity in the X-direction is approximately $52 \mathrm{KPa}$, while the corresponding maximum "load" in the Y-direction is $38 \mathrm{KPa}$. This has a significant bearing on the energy absorbing capability of the honeycomb (Gibson and Ashby[6]). Further work, such as the biaxial characterization reported in Chung and Waas[10], is needed to clearly ascertain the energy absorbing characteristics of these honeycombs under more general multiaxial loading situations.

\section{Concluding Remarks}

As a first step in understanding the crashworthiness of honeycomb sandwich panels, we have reported the results from a combined experimental and analytical study on the crushing response of circular celled polycarbonate honeycombs under uniaxial compressive loading in two mutually orthogonal directions. Our results show that hexagonally packed circular cell honeycombs are orthotropic (not transversely isotropic as would be expected). Initial cell ellipticity is found to be a major cause of this deviation from intended behavior. The induced orthotropy is reflected in various aspects of the crushing response. For example, the linear stiffness, the localized deformation pattern and the maximum load are all affected. Equivalent macroscopic characterizations of such honeycombs must reflect these observed experimental findings, since they bear heavily on our ability to accurately predict the energy absorbing characteristics of these honeycomb core materials and corresponding sandwich panels.

\section{Acknowledgement}

We are grateful to the Aerospace Engineering Department, University of Michigan, for financial support (JC). AMW is grateful to Prof. N. Triantafyllidis for bringing to his attention, in 1991, the early work by Gibson, Ashby and co-workers (references [2] and [5] in the bibliography).

\section{References}

1. A. N. Gent and A. G. Thomas, "Mechanics of foamed elastic materials" Rubb. Chem. Technol., Vol. 36, pp. 597, 1963.

2. L. J. Gibson, M. F. Ashby, G. S. Schajer and C. I. Robertson, "The mechanics of two dimensional cellular materials" Proc. $R$. Soc.,A 382, pp. 25, 1982.

3. M. J. Silva, W. C. Hayes and L. J. Gibson "The effect of non-periodic microstructure on the elastic properties of two-dimensional cellular solids" Int. J. Mech. Sci.,Vol. 37, pp 1161, 1995.

4. J. W. Klintworth and W. J. Stronge, "Elastoplastic yield limits and deformation laws for transversely crushed honeycombs" Int. $J$. Mech. Sci.,Vol. 30, pp. 273, 1988. 
5. L. J. Gibson and M. F. Ashby, "The mechanics of three-dimensional cellular materials" Proc. R. Soc.,A 382, pp. 43, 1982

6. L. J. Gibson and M. F. Ashby, "Cellular Solids: Structure and Properties" Pergamon Press, Oxford, 1988.

7. S. D. Papka and S. Kyriakides, "In-plane compressive response and crushing of honeycomb" J. Mech. Phys. Solids,Vol. 42, pp. 1499, 1994.

8. S. D. Papka and S. Kyriakides, "In-plane crushing of a polycarbonate honeycomb" Int. $J$. Solids Structures,Vol. 35, pp. 239, 1998.
9. Joachim L. Grenestedt, "Influence of wavy imperfections in cell walls on elastic stiffness of cellular solids" J. Mech. Phys. Solids,Vol. 46, pp. 29, 1998.

10. J. Chung and A. Waas, "Compressive response of circular cell honeycomb under in-plane biaxial stresses" UM Aerospace Engineering Department Report 98-3, Aerospace Engineering Department, University of Michigan, Ann Arbor, MI, 48109.

11. J. Chung and A. Waas, "The inplane elstic constants of a class of thin walled honeycombs" $U M$ Aerospace Engineering Department Report 991, Aerospace Engineering Department, University of Michigan, Ann Arbor, MI, 48109.

\begin{tabular}{|c|c|c|c|}
\hline $\begin{array}{c}\text { Aspect ratio } \\
\text { of PURC } \\
\text { a/b }\end{array}$ & $\begin{array}{c}\text { Analytical solution } \\
\text { of PURC } \\
E_{x}^{*}, E_{y}^{*}(\mathrm{KPa})\end{array}$ & $\begin{array}{c}\text { Numerical Simulations } \\
\text { of PURC } \\
E_{x}^{*}, E_{y}^{*}(\mathrm{KPa})\end{array}$ & $\begin{array}{c}\text { Difference } \\
\Delta E_{x}^{*}, \Delta E_{y}^{*}(\mathrm{KPa})\end{array}$ \\
\hline 1 & 751,751 & 752,752 & 1,1 \\
0.9596 & 701,816 & 702,817 & 1,1 \\
1.0427 & 806,693 & 802,694 & $-4,1$ \\
1.1129 & 898,603 & 896,604 & $-2,1$ \\
\hline
\end{tabular}

Table 1: Comparison of linear stiffnesses between analytcal solution and numerical simulation of PURC.

\begin{tabular}{|c|c|c|c|}
\hline Aspect ratio & $\begin{array}{c}\text { Analytical solution } \\
\text { of PURC } \\
E_{x}^{*}, E_{y}^{*}(\mathrm{KPa})\end{array}$ & $\begin{array}{c}12 \times 12 \text { imperfect cell } \\
\text {-numerical } \\
E_{x}^{*}, E_{y}^{*}(\mathrm{KPa})\end{array}$ & $\begin{array}{c}12 \times 12 \text { cell section } \\
\text {-experiment } \\
E_{x}^{*}, E_{y}^{*}(\mathrm{KPa})\end{array}$ \\
\hline 1 & 751,751 & 1178,699 & 1176,693 \\
0.9596 & 701,816 & 1178,699 & 1176,693 \\
1.0427 & 806,693 & 1178,699 & 1176,693 \\
1.1129 & 898,603 & 1178,699 & 1176,693 \\
\hline
\end{tabular}

Table 2: Comparison of linear stiffnesses for PURC results, full scale numerical simulations and experimental results. 


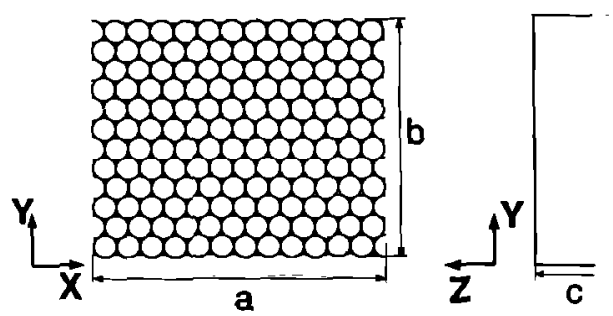

(a)

(b)

Figure 1: (a) Honeycomb specimens used for Tests under Compressive Loading. (b) Typical dimensions of the Test specimens.

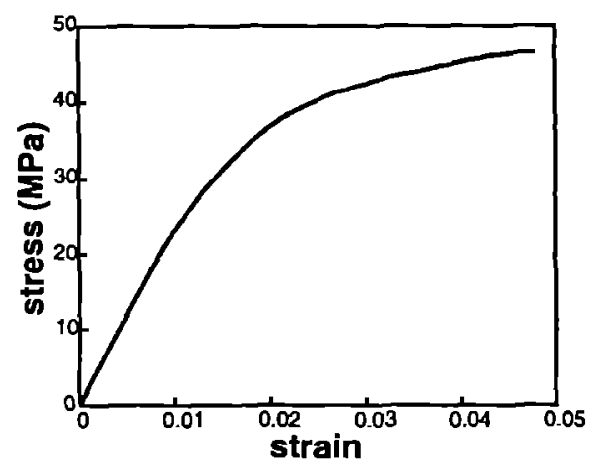

(a)

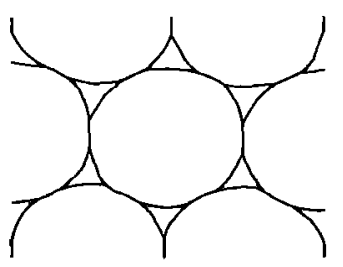

(b)

Figure 2: (a) The stress-strain curve for polycarbonate material. (b) A microsection of the honeycomb.
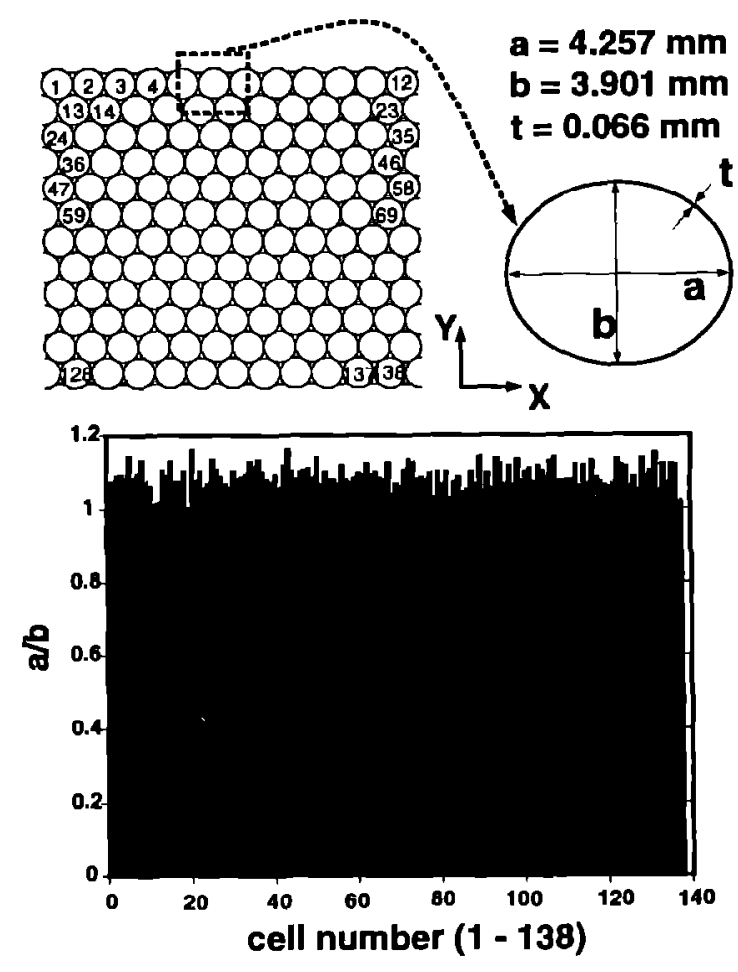

Figure 3: Deviation from circularity.

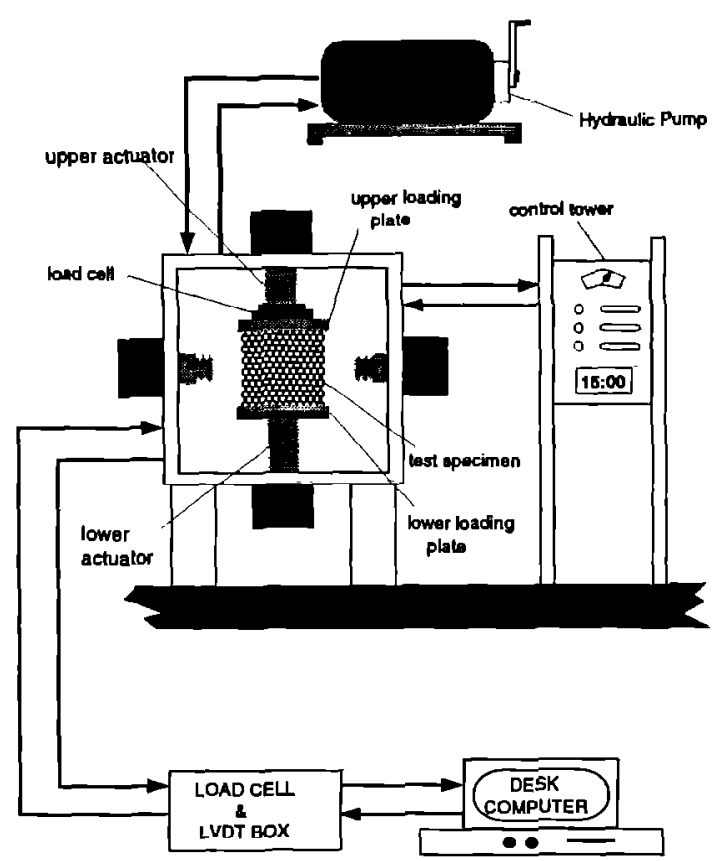

Figure 4: Schematic of test equipment used for inplane crushing of honeycomb 
(c) 1999 American Institute of Aeronautics \& Astronautics
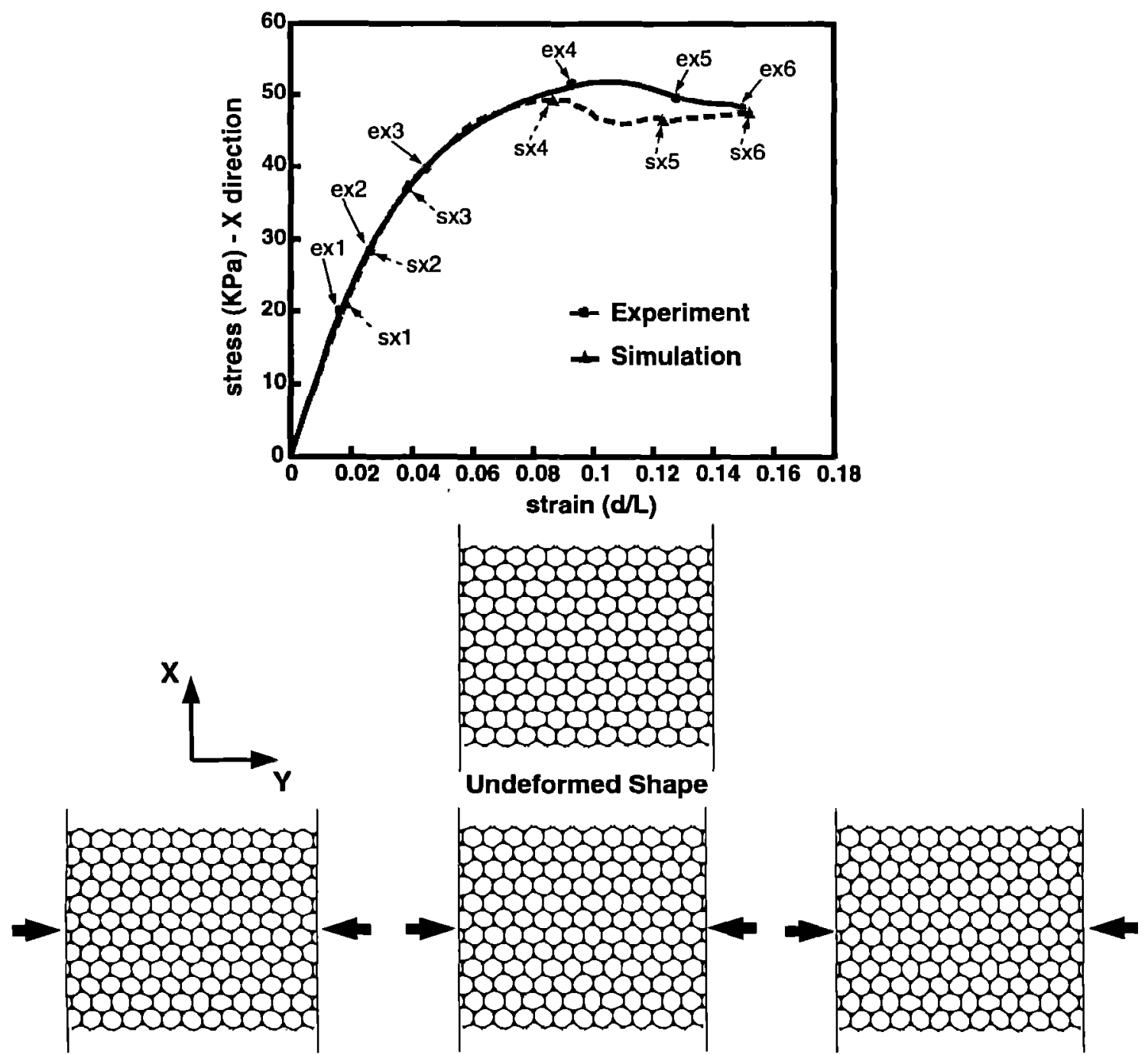

Deformed Shape : sx1

Deformed Shape : sx2

Deformed Shape : sx3

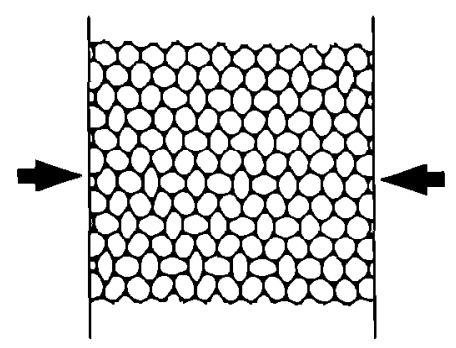

Deformed Shape : sx4

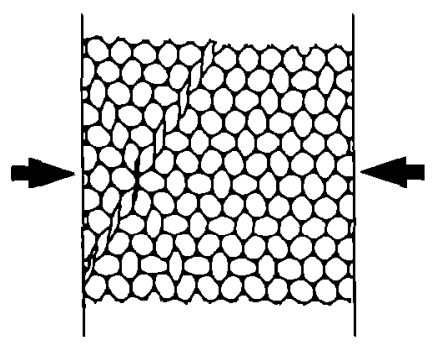

Deformed Shape : sx5

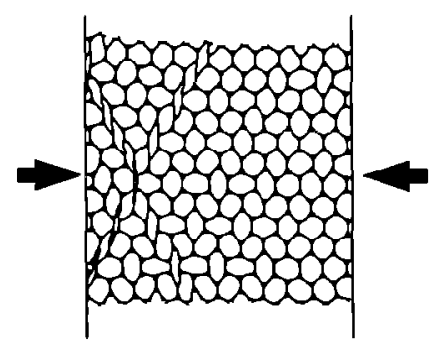

Deformed Shape :sx6

Figure 5: Stress-strain response under compressive loading in the $\mathrm{X}$-direction and a sequence of numerically simulated deformed honeycomb specimens under compressive loading in the $\mathrm{X}$-direction. 

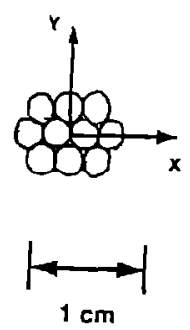

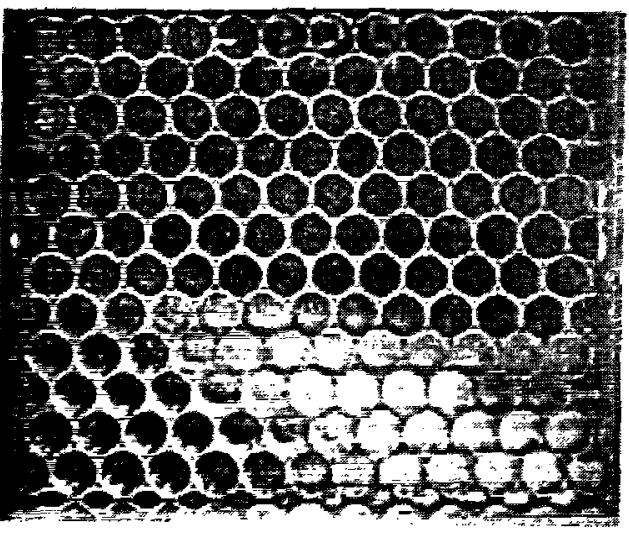

ex1

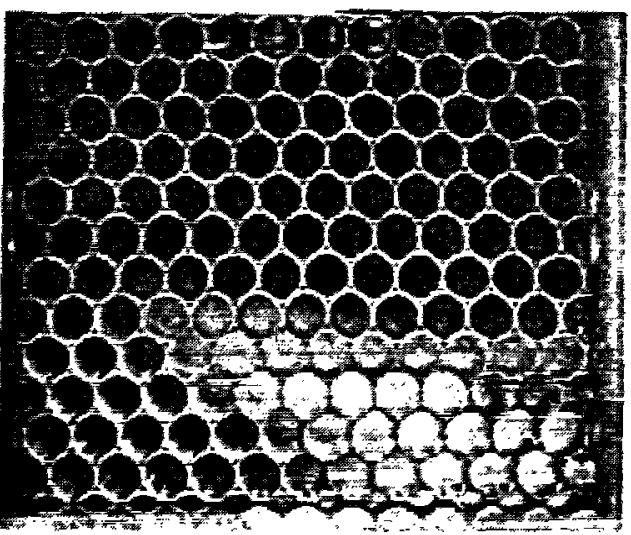

ex2

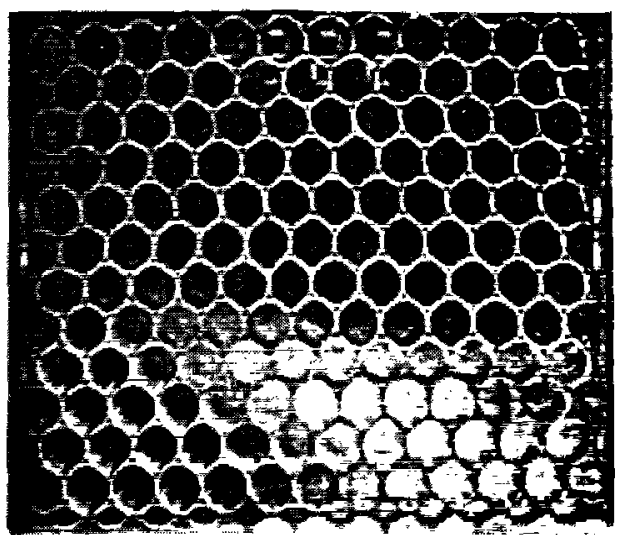

ex3

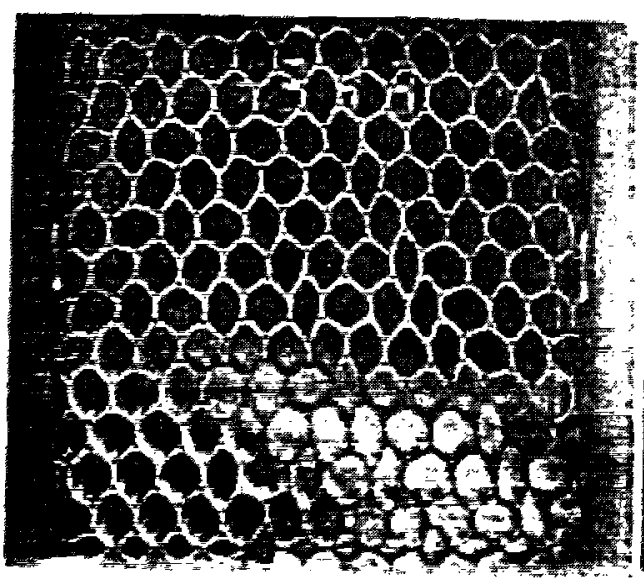

ex4

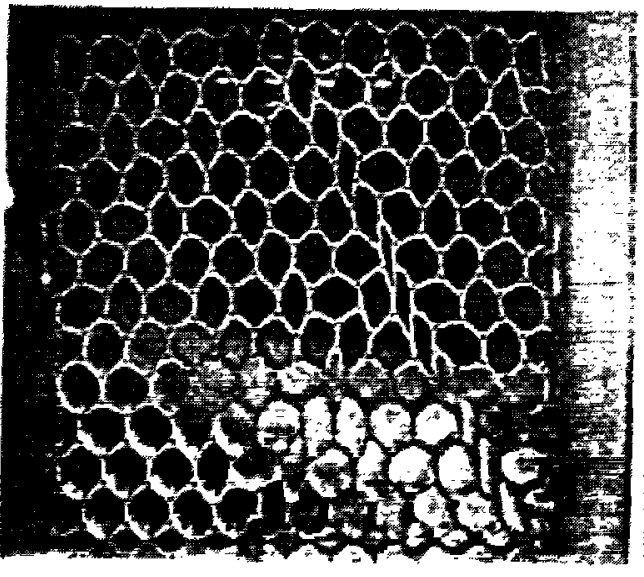

ex5

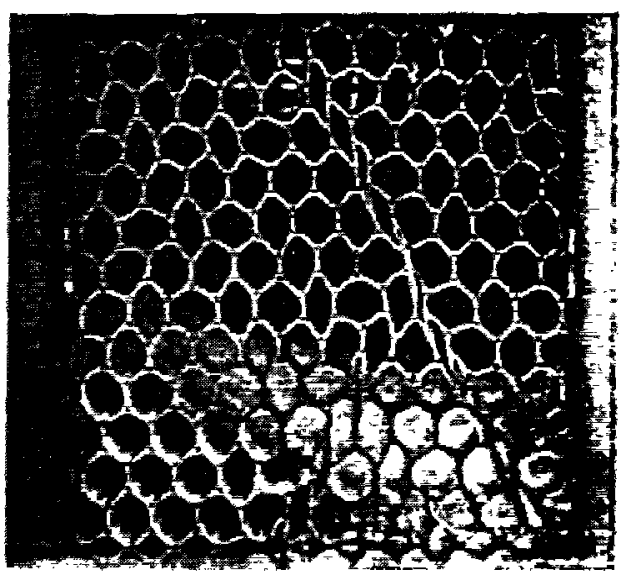

ex6

Figure 5: -continued; Stress-strain response under compressive loading in the X-direction and a sequence of photos of the deformed honeycomb specimen under compressive loading in the X-direction. 
(c) 1999 American Institute of Aeronautics \& Astronautics

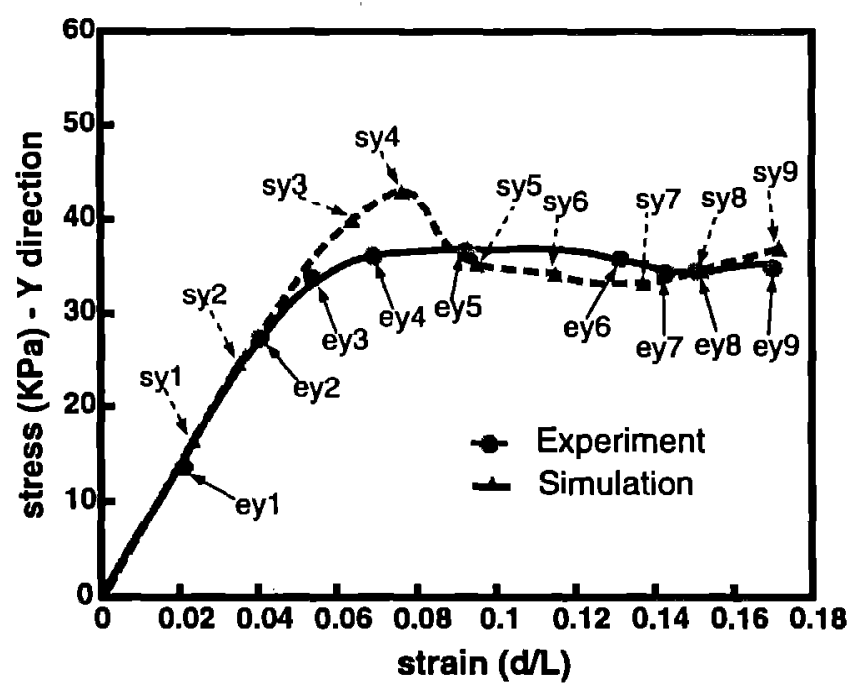

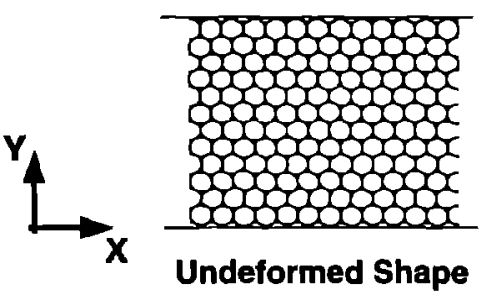
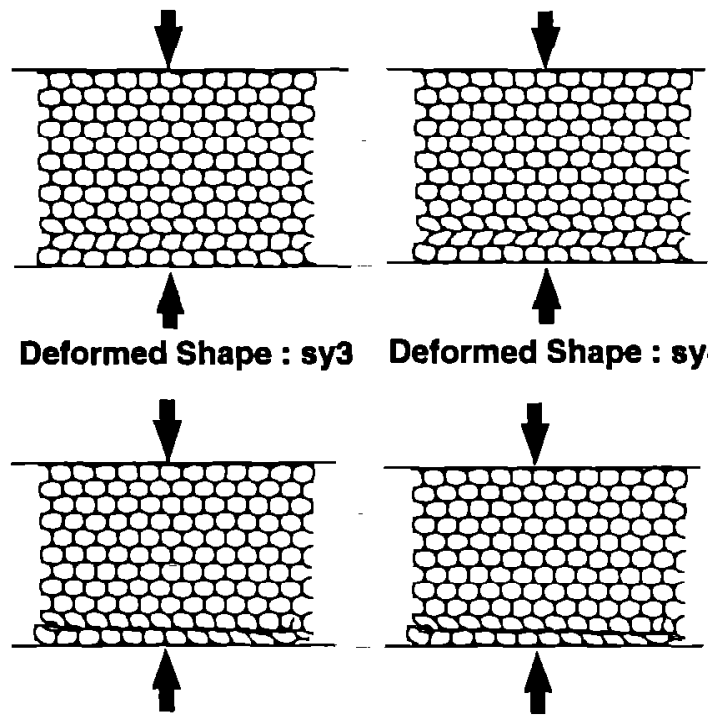

Deformed Shape : sy7

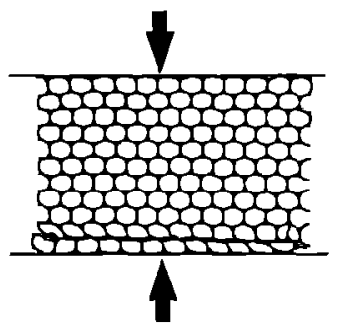

Deformed Shape : sy8

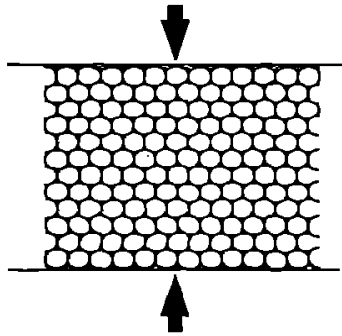

Deformed Shape : sy1

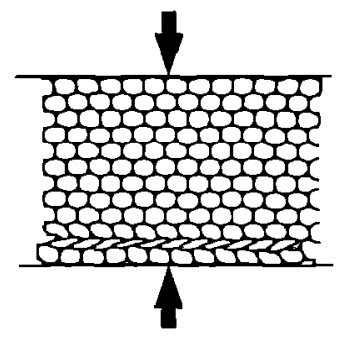

Deformed Shape : sy5

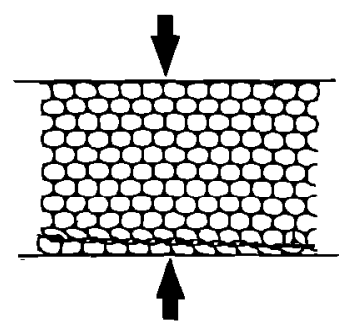

Deformed Shape : sy9

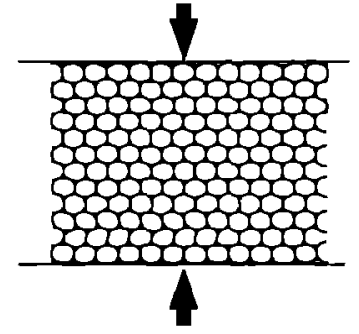

Deformed Shape : sy2

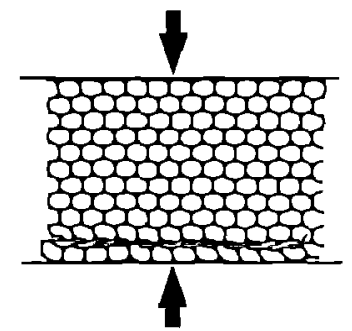

Deformed Shape : sy6

Figure 6: Stress-strain response under compressive loading in the Y-direction and a sequence of numerically simulated deformed honeycomb specimens under compressive loading in the Y-direction. 


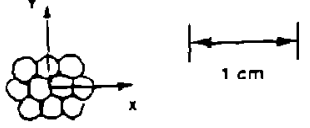

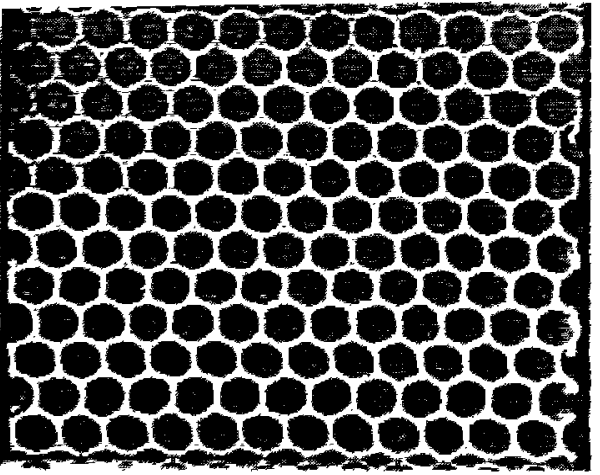

ey1

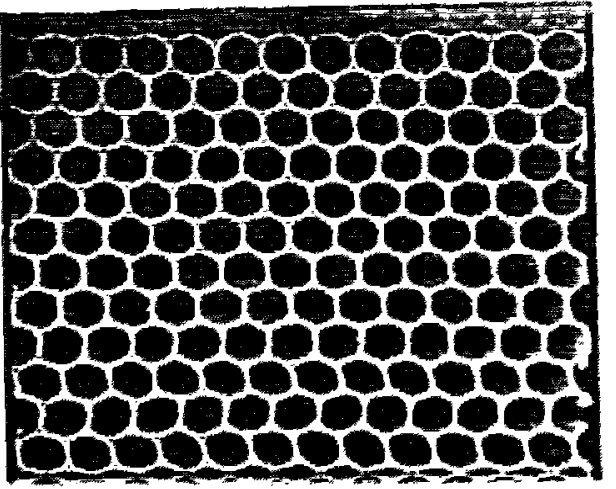

ey2

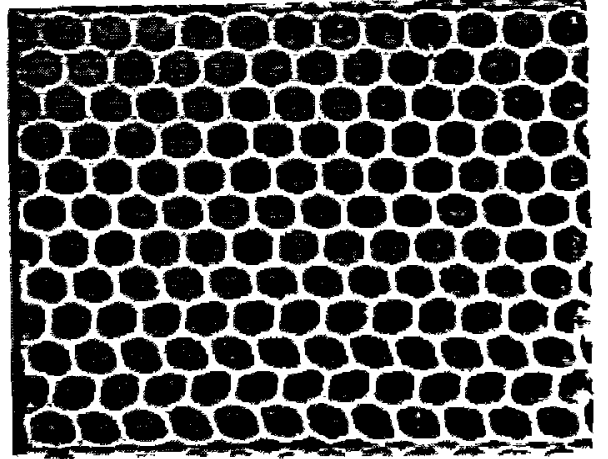

ey3

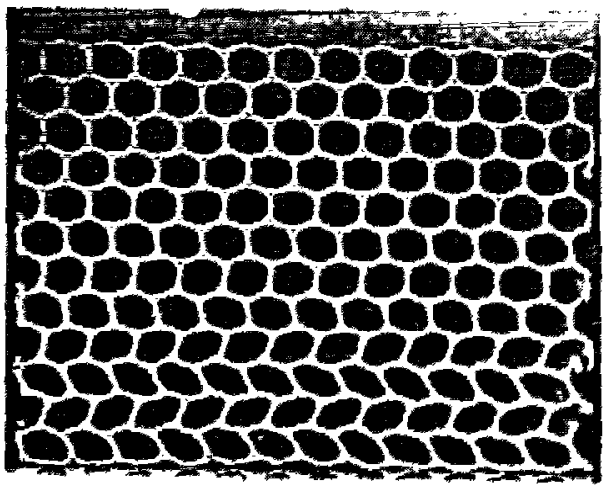

ey4

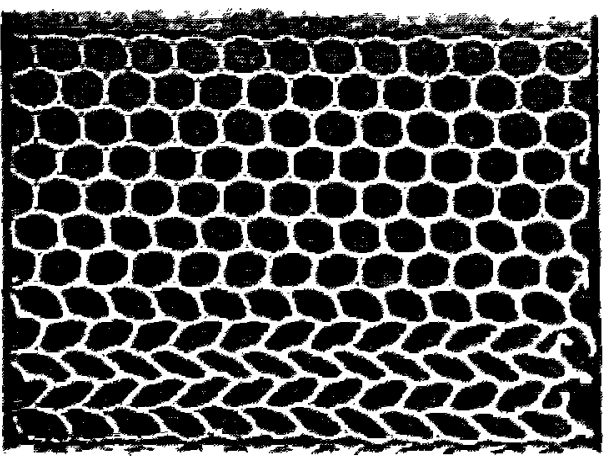

ey5

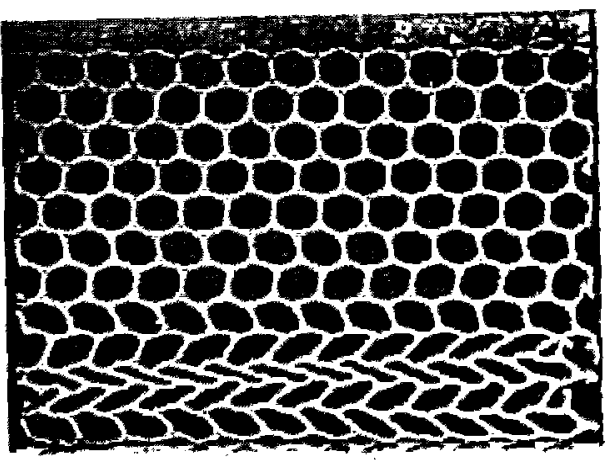

ey6

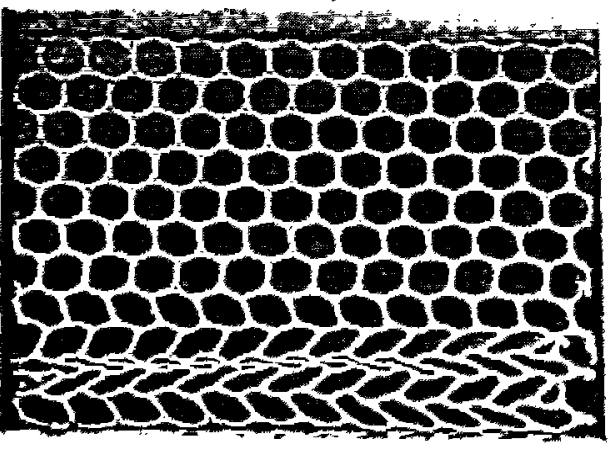

ey7

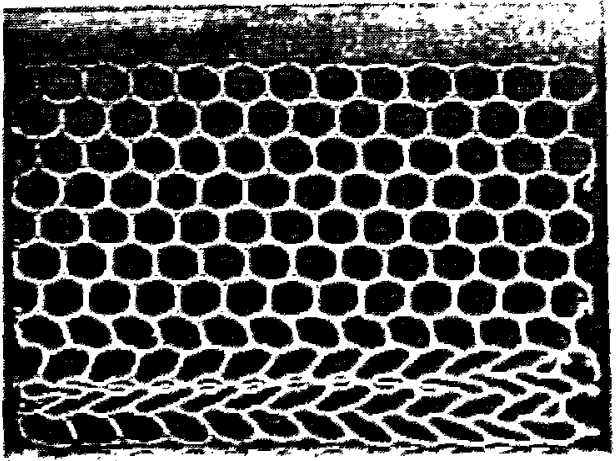

ey8

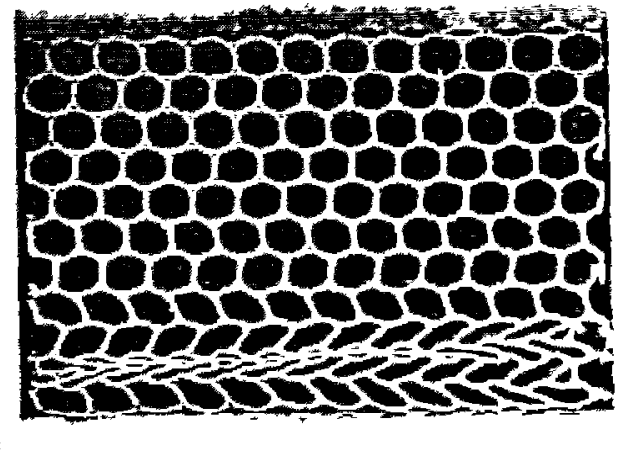

ey9

Figure 6: -continued; Stress-strain response under compressive loading in the Y-direction and a sequence of photos of the deformed honeycomb specimens under compressive loading in the $Y$-direction. 